\title{
Developing Patient-Refined Messaging for a Mailed Colorectal Cancer Screening Program in a Latino- Based Community Health Center
}

\author{
Jamie H. Thompson, MPH, Melinda M. Davis, PhD, LeAnn Michaels, BA, \\ Jennifer S. Rivelli, MA, Melissa L. Castillo, MSW, Brittany M. Younger, MSW, \\ Marta Castro, BA, Sacha L. Reich, MA, and Gloria D. Coronado, PhD
}

Introduction: Colon cancer is the second leading cause of cancer death in the United States, and screening rates are disproportionately low among Latinos. One factor thought to contribute to the low screening rate is the difficulty Latinos encounter in understanding health information, and therefore in taking appropriate health action. Therefore, we used Boot Camp Translation (BCT), a patient engagement approach, to engage Latino stakeholders (ie, patients, clinic staff) in refining the messages and format of colon cancer screening reminders for a clinic-based direct mail fecal immunochemical testing (FIT) program.

Methods: Patient participants were Latino, ages 50 to 75 years, able to speak English or Spanish, and willing to participate in the in-person kickoff meeting and follow-up phone calls over a 3-month period. We held separate BCT sessions for English- and Spanish-speaking participants. As part of the in-person meetings, a bilingual colon cancer expert presented on colon health and screening messages and BCT facilitators led interactive sessions where participants reviewed materials and reminder messages in various modalities (eg, letter, text). Participants considered what information about colon cancer screening was important, the best methods to share these messages, and the timing and frequency with which these messages should be delivered to patients to encourage FIT completion. We used follow-up phone calls to iteratively refine materials developed based on key learnings from the in-person meeting.

Results: Twenty-five adults participated in the in-person sessions (English [ $n=12]$; Spanish [ $n=$ 13]). Patient participants were primarily enrolled in Medicaid/uninsured (76\%) and had annual household incomes less than $\$ 20,000$ (67\%). Key themes distilled from the sessions included increasing awareness that screening can prevent colon cancer, stressing the urgency of screening, emphasizing the motivating influence of family, and using personalized messages from the practice such as 'I' or 'we' statements in letters or automated phone call reminders delivered by humans. Participants in both sessions noted the importance of receiving an automated or live alert before a FIT kit is mailed and a reminder within 2 weeks of FIT kit mailing.

Discussion: Using BCT, we successfully incorporated participant feedback to adapt culturally relevant health messages to promote FIT testing among Latino patients served by community clinics. Materials will be tested in the larger Participatory Research to Advance Colon Cancer Prevention (PROMPT) trial. (J Am Board Fam Med 2019;32:307-317.)

Keywords: Colon Cancer, Colorectal Cancer, Early Detection of Cancer, Hispanic Americans, Medicaid, Occult Blood, Patient Participation

Colon cancer is the second leading cause of cancerrelated death in the United States. ${ }^{1}$ In 2017, an estimated 135,000 persons will be diagnosed with

This article was externally peer reviewed.

Submitted 23 January 2018; revised 25 July 2018; accepted 30 July 2018.

See Related Article on Page 318. the disease and about 50,000 will die from the disease. ${ }^{2}$ Regular screening is effective in reducing the incidence and mortality of colon cancer by detecting precancerous polyps or cancer at early

From the Center for Health Research, Kaiser Permanente Northwest, Portland, OR (JHT, JSR, SLR, GDC); Oregon Rural Practice-based Research Network, Oregon Health \& Science University, Portland (MMD, LM); AltaMed Health Services, Los Angeles, CA (MLC, BMY, MC). 
curable stages. ${ }^{3}$ However, colon cancer screening rates are marked by pronounced demographic disparities, with Latinos, the uninsured, and those residing in the United States for fewer than 10 years having the lowest rates. ${ }^{4,5}$ Since these individuals typically receive care at 1 of over 1300 federally qualified health centers (FQHCs) nationwide, FQHCs are an ideal setting for interventions to increase screening rates and attenuate these disparities. ${ }^{6}$ One factor thought to contribute to the low screening rates among Latinos is low awareness of the need for screening and the limited availability of culturally appropriate patient health information. ${ }^{7,8}$ If patients are unable to understand a health condition and related screening options, they will likely experience difficulties engaging in meaningful conversation with a health care provider, choosing appropriate health action, or participating in recommended screening. ${ }^{9}$

Studies have shown that mailing fecal immunochemical test (FIT) kits directly to a patient's home (ie, direct mail) can increase colon cancer screening among FQHC populations. ${ }^{10-15}$ Among underserved patients whose screenings were not up-todate, direct-mail FIT outreach invitations resulted in significantly higher colon cancer screening compared with usual care. ${ }^{14}$ However, a recent systematic review found that while direct mail programs plus patient reminders were associated with higher FIT kit returns, studies provided limited comparative detail on optimal timing, content, or format of reminders. ${ }^{15}$

Authorship Contribution: Conception or design of the work: MMD, GD; Data collection: JHT, MMD, LM, JSR, MLC, BMY, MC, SLR, GDC; Data analysis and interpretation: JHT, MMD, LM, JSR, MLC, BMY, MLC, SR, GDC; Drafting the manuscript: JHT, MMD, GDC; Critical revision of the manuscript: JHT, MMD, LM, JSR, MLC, BMY, MC, SR, GDC; Final approval of the version to be published: JHT, MMD, LM, JSR, MLC, BMY, MC, SLR, GDC.

Funding: Research reported in this publication was supported by the National Institute on Minority Health and Health Disparities of the National Institutes of Health under Award U01MD010665. The content is solely the responsibility of the authors and does not necessarily represent the official views of the National Institutes of Health. Melinda Davis is supported in part by a Cancer Prevention, Control, Behavioral Sciences, and Populations Sciences Career Development Award from the National Cancer Institute (K07CA211971).

Conflict of interest: none declared.

Corresponding author: Jamie H. Thompson, MPH, 3800 N. Interstate Ave., Portland, OR (E-mail: jamie.h.thompson@ kpchr.org).
To address the need for culturally specific communications to improve FIT kit return rates in underserved populations served by FQHCs, and to understand patients' preferences for prompts, such as alerts (notifications occurring before FIT mailing) and reminders (notifications occurring after FIT mailing), to a direct-mail program, we implemented Boot Camp Translation (BCT). BCT is a patient engagement approach to turn complex medical concepts and guidelines into locally relevant health messages and formats that are understandable and meaningful to patients. ${ }^{9}$ The first stage of our Participatory Research to Advance Colon Cancer Prevention (PROMPT) study employed BCT in the development of interventions for a pilot comparative effectiveness trial. Our BCT process was designed to answer 2 primary research questions: 1) What should we emphasize in our colon cancer screening messages to patients? and 2) What is the optimal timing and modality for delivering reminders to a FIT program? This article describes the BCT process and summarizes our findings.

\section{Methods}

\section{Setting and Participants}

The PROMPT study is a collaboration between 2 research institutions (Kaiser Permanente Center for Health Research and Oregon Health \& Science University) and a large independent FQHC in California with 27 medical clinics serving 280,000 patients. The majority of the FQHC's patients are Latino $(82 \%)$, and almost $40 \%$ of these Latino patients prefer speaking Spanish. Oregon Health \& Science University's Oregon Rural Practice-based Research Network provided BCT expertise. PROMPT was approved by the Institutional Review Board of Kaiser Permanente Northwest (Portland, OR), with ceding agreements from relevant institutions. Due to the minimal risk of the BCT process, the requirement for informed consent was waived. The study is registered on ClinicalTrials.gov (NCT03167125) and the full study protocol is available elsewhere. ${ }^{16}$

BCT, originally developed by researchers at the Colorado High Plains Research Network, is a method for engaging stakeholders in translating health information into ideas, messages and materials that resonate with target community members. ${ }^{9,17}$ The approach unites community mem- 
bers, researchers, and medical experts to address local health concerns. ${ }^{18}$ BCT uses a consensusbuilding process that incorporates an iterative schedule of in-person meetings combined with short, focused phone calls. BCT generally addresses 2 main questions: "What do we need to say in our message?" and "How do we deliver that message?" The typical BCT process requires about 20 to 25 hours of participant time over 4 to 18 months depending on the scope of the project and complexity of the health topic. ${ }^{9,19}$ BCT facilitators in this study were experienced in facilitating patient engagement activities with diverse populations, had received BCT facilitator training (see www.bootcamptranslation.org), and had either participated in or led prior BCT sessions. We adapted the BCT approach used in our study because we were focused on improving timing of reminder delivery and refining existing message content, ${ }^{20,21}$ rather than creating new messages or delivery methods. In our adapted BCT process, we asked individuals to participate in a 1-day in-person session and 3 follow-up group phone calls over a period of 3 months. BCT activities occurred between May 12 to July 12, 2017.

\section{Study Procedures}

We conducted 2 parallel BCT sessions: 1 in English and 1 in Spanish. The PROMPT BCT research team comprised a study principal investigator, 2 English- and 2 Spanish-speaking BCT facilitators, and 4 project personnel. The Englishspeaking BCT facilitators attended a 2-day BCT training session delivered by experts from the Colorado High Plains Research Network and trained the Spanish-speaking BCT facilitators, including providing a manual for review and discussion, and observing the Spanish-language BCT onsite to support the facilitators. Recruitment and BCT materials were developed in both English and Spanish, and separate in-person and phone sessions were held for English- and Spanish-speaking participants based on individual language preference. Using a convenience sampling method, FQHC clinic staff at 2 pilot clinics aimed to recruit 12 patient participants for each BCT cohort. Eligible patient participants were Latino, ages 50 to 75 years (the age range for colon cancer screening ${ }^{3}$ ), able to speak English or Spanish, and willing to participate in the in-person meeting and follow-up group phone calls. Two clinic staff were asked to partic- ipate in the BCT process, and 2 bilingual members of the PROMPT advisory board observed the sessions.

We used BCT to identify important themes and preferred messages for written and verbal communications about colon cancer screening; select optimal timing and frequency of delivering reminders for the direct mail program; refine selected messages; and define the components of the intervention for the PROMPT pilot. An overview of the PROMPT BCT is presented in Figure 1 and summarized below.

\section{Data Collection and Analysis}

\section{In-Person Session}

The purpose of the in-person session was to collectively identify important themes from existing colon cancer screening communications $s^{20,21}$ and select optimal timing and mode of delivering screening reminders. The 6-hour meeting started with 2 presentations by a bilingual national colon health expert. Each presentation lasted 1 hour; the first was a scientific medical presentation addressing colon health ${ }^{5}$ and the second focused on effective colon health messages to improve Latino screening participation. ${ }^{21}$ Expert presentations are an integral element of BCT in that they empower participants to become experts on the topic and ultimately a voice for their community. ${ }^{9}$ Following each expert presentation, trained BCT facilitators led brainstorming sessions with attendees to identify salient colon cancer screening messages.

In the afternoon, 3 interactive small group sessions ("breakout stations") staffed by a facilitator and notetaker were held to obtain more detailed input on 1) incorporating community appropriate messages into existing FIT reminders, and 2) selecting optimal modality, timing, and frequency of these prompts. Station 1 displayed sample FIT kit mailing materials obtained from existing programs. Station 2 provided sample text message reminders, patient portal messages, letters, and automated phone call recordings. Station 3 addressed the timing with which reminder messages should be delivered. At this station, participants were asked to propose a plan for timing and mode of reminder delivery on worksheets using stickers indicating reminder modality. A sample of a completed worksheet is shown in Figure 2. The research team collected completed worksheets and transferred results to a $4^{\prime} \times 6^{\prime}$ wall-size poster in the meeting 
Figure 1. Boot camp translation overview. FIT, fecal immunochemical test.

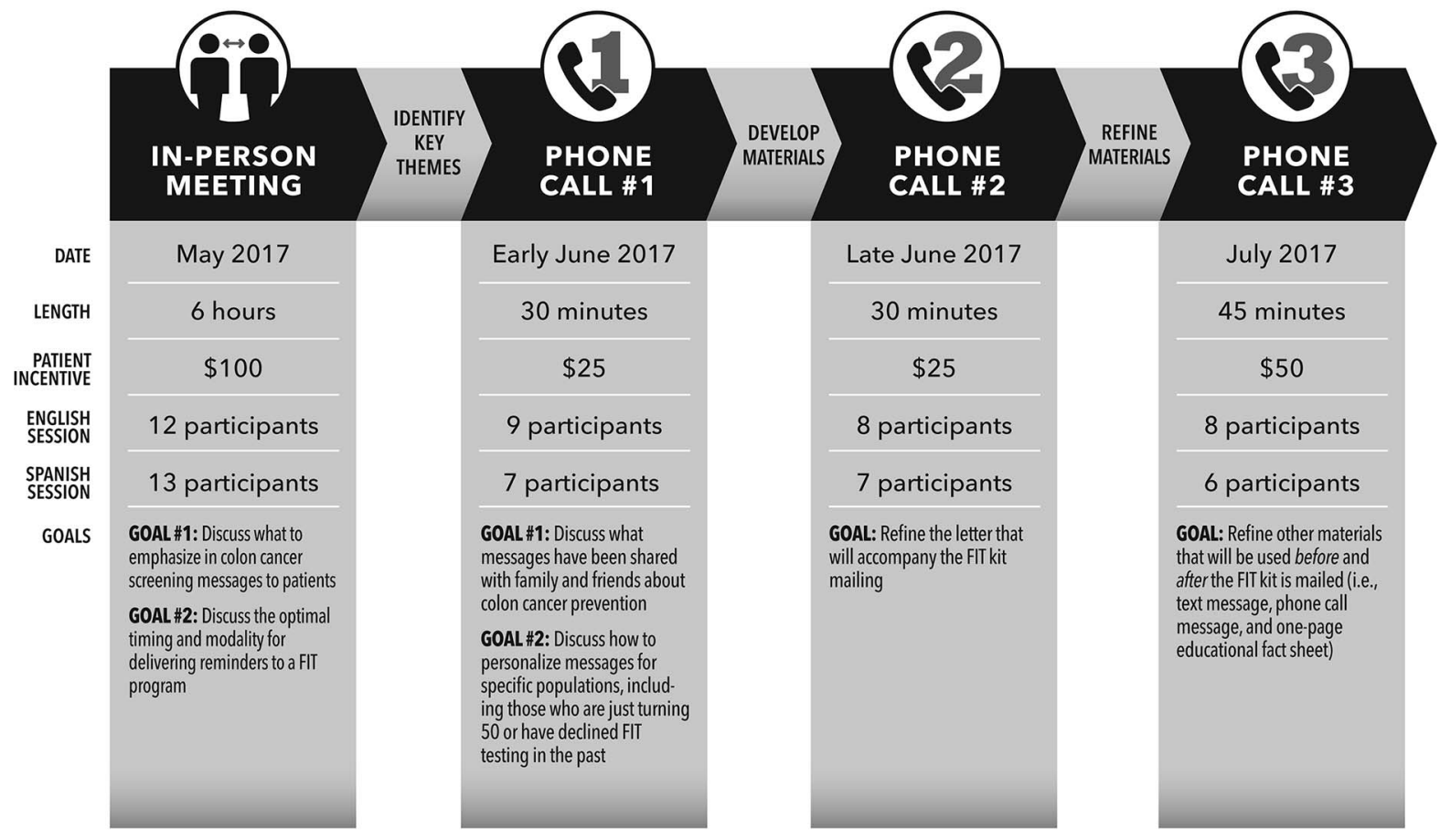

room to identify and discuss trends with the larger group.

Before the session ended, participants completed a brief voluntary questionnaire about their colon cancer screening history, health literacy status, cell phone usage, and demographics. Attendees also completed an evaluation form about their BCT experience. Questions were presented using a 1 to 5 -point Likert Scale (5 = strongly agree) that focused on facilitator skill, session utility, group comfort level, and overall sense of accomplishment.

Field notes were taken by at least 2 research team members during each in-person session. After the meeting, the research team gathered to debrief. This session was led by the BCT facilitators and lasted 2 hours. The goals of the debrief were to identify key themes with respect to important screening messages and ideal timing and mode of reminder delivery, as well as determine topics to be explored during the follow-up phone calls. Input from both the English- and Spanish-speaking participants were considered to determine if and how messages might vary based on language group. Findings informed the development of draft PROMPT study materials that were reviewed by participants during the group phone calls described below.

\section{Follow-Up Phone Calls}

At the conclusion of the in-person session, participants voted on and selected the time frame for the follow-up phone calls to optimize attendance. The 3 group calls, ranging in duration from 30 to 45 minutes, were designed to refine the English- and Spanish-language PROMPT materials that were developed based on key findings from the in-person meeting and input from each call. Each phone call started with a brief review of the objectives of the discussion, clarifications from the previous meeting, then a facilitated review of updated draft materials mailed to participants before the scheduled calls. Field notes were taken by the research team during each call and used to help refine the materials. Call 1 focused on participants discussing how they shared their newfound knowledge from BCT with their family and friends, and how messages should be personalized for different groups of patients, such as those who had declined FIT testing in the past. Calls 2 and 3 focused on participant input and revisions to draft materials including an introductory letter, reminder messages and scripts, and an educational brochure. Following the series of 3 phone calls, researchers finalized the materials for implementation during the comparative effectiveness pilot study. ${ }^{16}$ 
Figure 2. Participant worksheet for timing and mode of reminders. FIT, fecal immunochemical test.

\section{Worksheet}
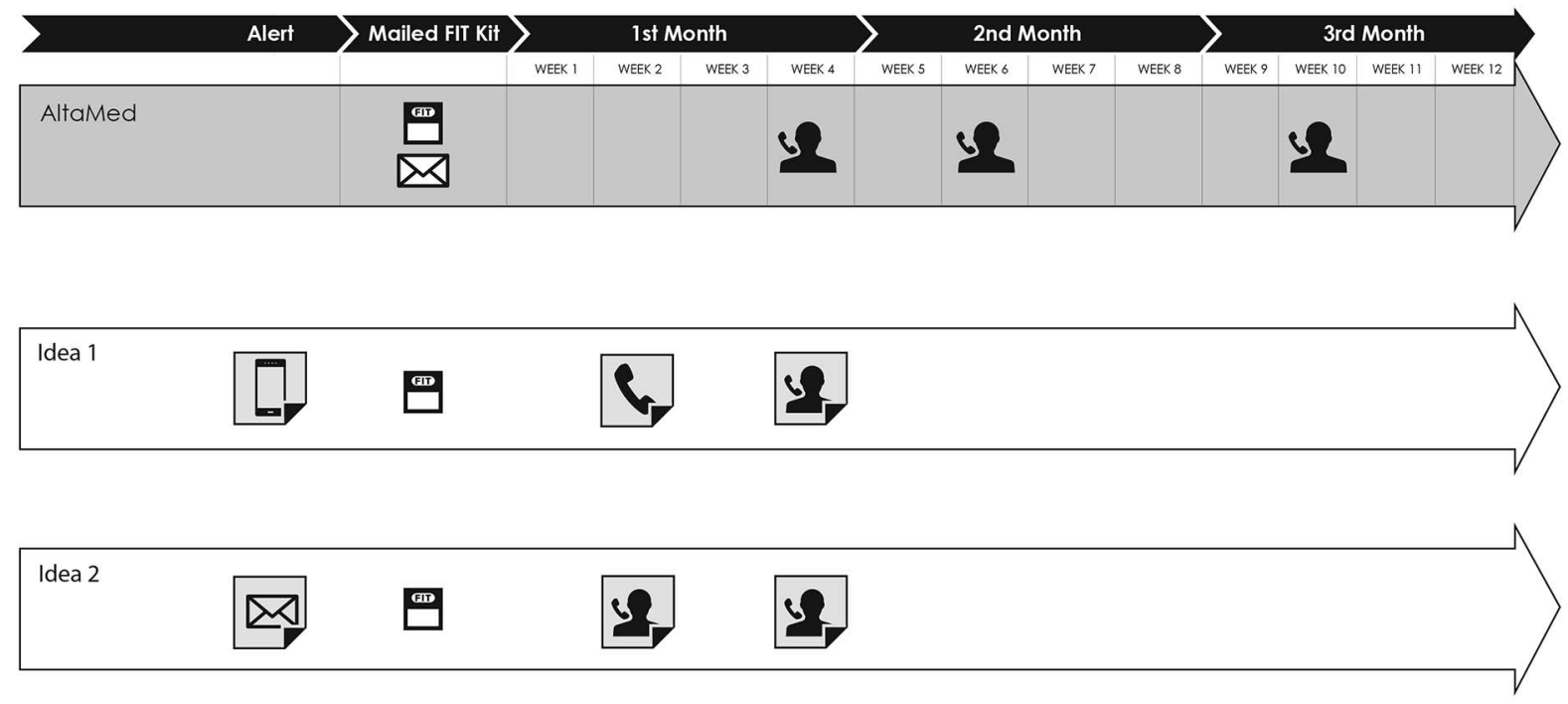

\section{PRÓMPT}

Get tested today! ¡Hágase la prueba, hoy!

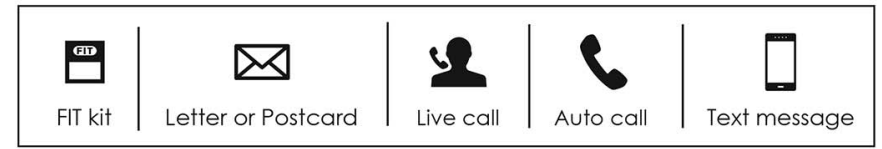

$60008 \mathrm{~B} 10 / 17 \mathrm{CHR}$

\section{Results}

\section{Study Population}

Twenty-five adults participated in the 2 BCT inperson sessions (12 in English language; 13 Spanish language) (Figure 1). An estimated 54\% (Spanish session) to $75 \%$ (English session) of these attendees took part in the follow-up phone calls. Figure 1 shows details of the duration of the in-person session and follow-up phone calls, as well as number of participants at each stage. As summarized in Table 1 , the majority of the participants were clinic patients (84\%); 4 were medical assistants from the FQHC. Compared with the patient participants in the English language session, those in the Spanish session were more likely to be female ( $91 \%$ vs $60 \%$ ), uninsured ( $36 \%$ vs $0 \%$ ), have an annual household income less than $\$ 20,000$ ( $82 \%$ vs $50 \%$ ), have less than a high school education $(73 \%$ vs $20 \%$ ), be born outside of the United States (100\% vs $20 \%$ ), request assistance to read health information provided by their doctor or pharmacy ( $82 \%$ vs $40 \%)$. Overall, participants were more likely to use their cell phone to send or receive texts than emails (English, $60 \%$ vs $40 \%$; Spanish, $82 \%$ vs $18 \%$ ).

\section{Key Themes}

\section{Education and Messaging}

Participants in both English and Spanish sessions identified key factors that could motivate patients to complete colon cancer screening. Participants noted that it was important to raise patient awareness about colon cancer and prevention and stress the importance of getting screened. Effective messages were those that emphasized family as a motivator for screening, and that were personalized from the doctor's office to the patient. Themes and relevant participant quotes are outlined in Table 2 and summarized below.

First, participants discussed the need for more education about colon cancer prevention. In general, there was an initial lack of knowledge about colon cancer screening-including at what age patients should be screened and that screening was not just for "those who already had cancer." This awareness gap was overcome during the expert presentation and prompted an interest in learning more about colon health and screening, and opportunities to share that information with friends and family. Patients desired education in a variety of 
Table 1. Characteristics of English and Spanish Language Boot Camp Translation Participants, May 12 to 13, $2017^{*}$

\begin{tabular}{lcc}
\hline & English-Language Session & Spanish-Language Session \\
\hline Number of participants (in-person meeting) ${ }^{\dagger}$ & $\mathbf{1 2}$ & $\mathbf{1 3}$ \\
Number of patients & 10 & 11 \\
Number of clinic staff & 2 & 2 \\
Boot Camp Translation patients only & 57.1 & 56.6 \\
Age (years), mean & 60 & 91 \\
Female, \% & 80 & 100 \\
Latino, \% & 80 & 36 \\
Medicaid, \% & 0 & 36 \\
Uninsured, \% & 50 & 82 \\
Annual household income less than \$20,000, \% & 20 & 73 \\
Less than high school education, \% & 20 & 100 \\
Born outside of the United States, \% & 40 & 82 \\
Sometimes/often/always need help to read materials & & 82 \\
$\quad$ from their doctor or pharmacy, \% & 60 & 18 \\
Use cell phone to send or receive texts, \% & 40 & \\
\hline
\end{tabular}

${ }^{*}$ We did not test for statistical significance given the small sample size of the two groups.

${ }^{\dagger}$ This participant characteristics table does not include the Advisory Board members who observed the English- and Spanish-language sessions.

formats including 1-on-1 education from their providers, informational fact sheets, and health education classes.

Participants identified 3 key areas to emphasize in messaging around screening. First, the concept of getting tested early as an action to prevent colon cancer or treat early forms of colon cancer resonated strongly with the BCT participants. They supported messaging that stressed the timely completion of the FIT test: "Getting screened is part of your health care. This is your chance to play doctor. Just do it!" "Complete the test today-it could save your life!" Second, the group underscored the power of using family-oriented messages or personal stories as a motivator to get screened. Messages about taking action to stay healthy for children and especially grandchildren were strong motivators for the attendees: "Take control of your health. You and your family will be happy you did!" Finally, BCT participants emphasized the importance of receiving personalized messages from providers, achieved through "I" or "we" statements in letters and automated phone call reminders that stated the patient's name and are delivered by a human voice. The FQHC preferred using terminology such as "we" or "your health care team" to emphasize the coordinated aspect of care delivered within their network.
In addition to these educational messages, participants in the Spanish-speaking group identified additional themes including the importance of providers anticipating patient barriers. Spanish-speakers noted the importance of establishing "compromiso" (commitment) and "confianza" (trust) between patients and providers to tackle barriers before the patient becomes noncompliant. Similar to the English-speakers, they emphasized the importance of providers relaying colon cancer information in "clear and simple terms" that they could understand and share with their family. Prevention was also a critical theme in both language groups and the need to get screened before symptoms seem resonated strongly with the Spanish-speaking group where typical behavior is often to wait until symptoms seem before seeing a provider.

\section{Timing and Frequency of Reminders}

Through BCT activities, we learned that patients generally wanted to 1) receive some type of notification about the FIT kit before it is mailed, and 2) receive a reminder within 2 weeks of FIT kit mailing. Although we planned to focus on the timing and frequency of reminders, we learned that many participants desired an alert (notification before FIT mailing) about the FIT test arrival, so they would 1) expect it, and 2) understand the importance of 
Table 2. PROMPT Boot Camp Translation Themes and Participant Quotes

\begin{tabular}{|c|c|}
\hline Themes & Quotes \\
\hline \multirow{5}{*}{$\begin{array}{l}\text { Increase patient awareness about colon cancer } \\
\text { and prevention }\end{array}$} & "People need to know that you can prevent colon cancer." \\
\hline & $\begin{array}{l}\text { "Yo no sabia de esta prueba, que bueno, podemos decirle a las } \\
\text { amistades y familias." [I did not know about this test, great, we can } \\
\text { tell friends and families.] }\end{array}$ \\
\hline & $\begin{array}{l}\text { "Yo no tenía conocimiento de esto, yo decía esto es para viejitos pero } \\
\text { abora sé que no hay edad." [I had no knowledge of this, I thought } \\
\text { this was for old people but now I know there is no age. }]\end{array}$ \\
\hline & $\begin{array}{l}\text { "Yo no sabia, que cumpliendo los } 50 \text { años que se tenia que bacer esta } \\
\text { prueba." [I did not know that when I turned 50, I had to do this } \\
\text { test. }]\end{array}$ \\
\hline & $\begin{array}{l}\text { "En general nosotros los Latinos vamos al médico para que nos curen y } \\
\text { no para prevenir." [In general, we Latinos go to the doctor to be } \\
\text { cured and not to prevent.] }\end{array}$ \\
\hline \multirow[t]{2}{*}{ Stress importance of screening } & $\begin{array}{l}\text { "If you want me to do it, call me next week. If you wait until } 4 \\
\text { weeks, I'll have already thrown it away!" }\end{array}$ \\
\hline & "It's important to get tested, so just get it done." \\
\hline \multirow[t]{2}{*}{ Emphasize family as a motivator for screening } & "Talking about families is important and a good motivator." \\
\hline & "Do it for your children and grandchildren." \\
\hline \multirow[t]{2}{*}{ Use personalized messages } & "I want to get a letter from the doctor." \\
\hline & "I like the personal connection. Treat me like a buman being." \\
\hline \multirow[t]{2}{*}{$\begin{array}{l}\text { Encourage medical staff to use clear and } \\
\text { simple terms when introducing colon } \\
\text { cancer screening }\end{array}$} & $\begin{array}{l}\text { "El medico nos dice pero en términos médicos y no entendemos- yo no } \\
\text { sabia lo que era." [The doctor tells us but in medical terms and we } \\
\text { do not understand-I did not know what it was.] }\end{array}$ \\
\hline & $\begin{array}{l}\text { "Varias veces me han mandado esta prueba y no la be becho, pero } \\
\text { abora si." [Several times I bave been sent this test and I bave not } \\
\text { done it, but now I will.] }\end{array}$ \\
\hline
\end{tabular}

PROMPT, Participatory Research to Advance Colon Cancer Prevention trial.

completing the test. Most participants preferred to be reminded earlier than the 4 weeks postmailing that had been standard practice at the FQHC. One participant noted, "If you want me to do the test, remind me the first or second week. If you remind me 1 month later, I will have lost the test!" Spanish speakers also mentioned that getting a reminder to complete the kit indicated the clinic cared about their health, and a wait of 4 weeks implied that the test was not important. Participants were open to different reminder formats, including automated or live calls, but tended to prefer live calls because of the personal connection and ability to follow up with clinic staff if there were questions.

\section{Drafted Materials Refined through BCT Follow-Up Calls}

Through the series of iterative phone conversations, BCT participants refined bilingual PROMPT materials, including an introductory letter included in the FIT kit mailing, a text message alert, an automated call reminder, and an educational brochure. Final materials are described below and available at www. mailedfit.org. For the sake of consistency, the FQHC also refined their live call script to reflect themes and terminology that emerged from BCT.

\section{Introductory Letter Included in the FIT Kit Mailing}

Revisions recommended and approved by BCT participants included 1) the addition of details about colon cancer and the need for prevention and early action, 2) emphasis on a free test, 3) inclusion of a photograph of a multigenerational Latino family, 4) the addition of a colon diagram for visual appeal and education, and 5) pointed messages about how screenings can save lives and the importance of taking control of one's own health. The group wanted more emphasis on the test being "simple" and "something you can do at home," and to include a message about family such as "Get your screening done so you can live a healthy life with your family." Figure 3 presents the final enhancements made to the original introductory letter in response to the BCT phone call discussion.

\section{Text Message Alert}

To reduce the overall number of automated phone calls patients received, the research team opted to 


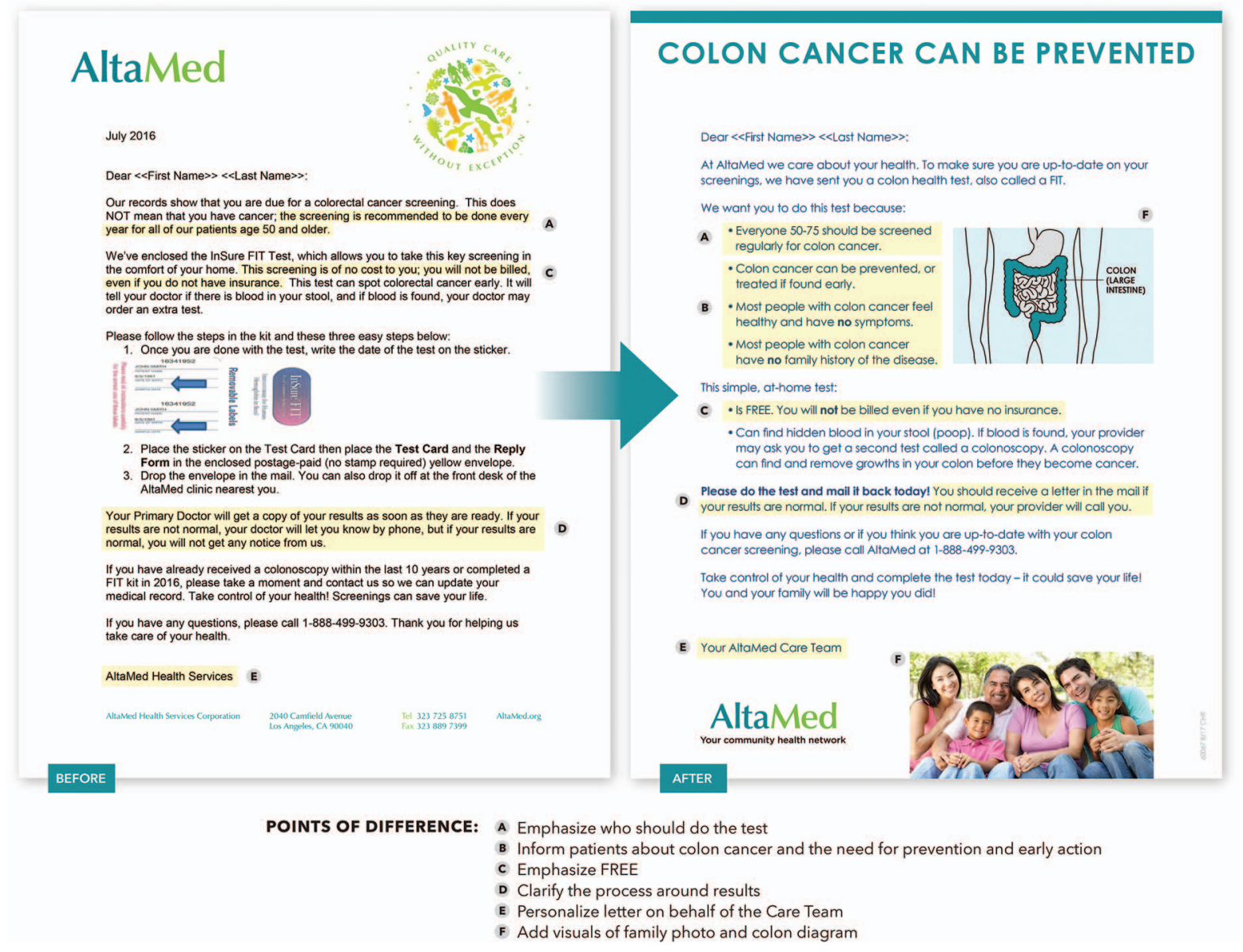

send the alert message via text. The final text alert message was limited to the 140 -characters specified by the phone vendor and is provided below:

[FQHC] is mailing you a free colon health test to do at home.

Complete the test today-it could save your life!

Questions? Call [number].

The text message succinctly highlighted BCT themes: the test is free, can be done at home, and is important to complete.

\section{Automated Phone Call Reminder}

Continuity of messaging was stressed by BCT participants. So, too, the final automated phone call script emphasized the test was free, could be completed at home, and highlighted the importance of testing. The family message was added at the end of the call script:

Hello [Patient First Name], I am calling from [FQHC] to remind you to complete the free colon bealth test we sent you. Take control of your health and complete the test today. You and your family will be happy you did!

To personalize the message, the automated phone call was recorded using a human voice instead of a robotic voice, and the patient name was spoken in the recording.

\section{Educational Brochure}

After learning from BCT participants that there was a desire for increased education about colon cancer and screening, we developed a tri-fold brochure using participant-preferred messages and simple infographics from the BCT expert presentation. Overall, the patient participants endorsed the use of an educational brochure and asked for simplified content for ease of understanding. As a result of this feedback, the research team condensed the information into a 2-sided bilingual fact sheet, which presented informative statistics about 
colon cancer diagnoses and deaths, answered the following questions: "What is colon cancer? When should I get tested? How do I get tested?” and noted the FIT test is free.

\section{Participant Experience}

Summary scores from the evaluation forms for both the English and Spanish sessions indicated strong support of, and positivity toward, the BCT inperson session. Average scores ranged from 4.7 to 4.9 and 4.6 to 5.0 for the English and Spanish sessions, respectively. We heard from attendees that they learned useful information about colon health and FIT testing, felt respected throughout the process by facilitators and other participants, and experienced a sense of accomplishment. Overall, BCT participants demonstrated strong levels of engagement throughout the in-person session as reflected by the constant flow of questions and conversations throughout the meeting.

\section{Discussion}

Using BCT, we successfully incorporated participant feedback to 1) adapt culturally relevant health messages, and 2) refine the timing and modality of prompts to promote FIT completion among Latino patients served by community clinics. Specifically, participants valued messages that increased awareness about colon cancer and prevention, stressed the importance of screening, emphasized the motivating influence of family, and used personalized statements such an "I" or "we" in letters or automated phone call reminders delivered by human voices. In addition, both the English- and Spanish-speaking sessions noted the importance of patients receiving an automated or live alert before a FIT kit is mailed and a reminder within 2 weeks of FIT kit mailing. We used key findings from the BCT process to refine bilingual PROMPT materials, including an improved introductory letter included in the FIT kit mailing, a text message alert, an automated call reminder, and an educational fact sheet.

BCT provided the ideal platform to engage patients in an interactive process to adapt colon health information into locally relevant and meaningful messages on a screening reminder schedule accessible to this population. When patients understand a health condition and the need for screening, they become advocates for their own health, par- ticipate in conversations with their provider, and adhere to recommended screening measures. ${ }^{3}$ The delivery of an expert presentation early in the BCT process provided participants with the opportunity to become knowledgeable about the health topic and consequently empowered them to take an active and informed role in discussions about messaging. This educational component allowed the entire group to identify common language to have an effective conversation about colon health and colon cancer prevention. It also highlighted that despite current evidence-based recommendations for screening, some participants recommended that screening should start earlier-and that swift completion of screening should be articulated.

\section{Lessons Learned}

The typical structure of BCT includes face-to-face meetings and short, focused phone calls over 6 to 12 months. ${ }^{9}$ We modified the original BCT approach because our study focused on refining existing message content and improving timing of reminder delivery, rather than creating new messages. In our shortened BCT process, we asked individuals to participate in a 1-day in-person session and 3 follow-up group phone calls over a period of 3 months. While our rapid timeline did keep participants engaged in the process, it limited the amount of time available for participants to review materials before the phone calls. In addition, many patient participants had limited access to, and experience with, technology, so refining materials by conference call provided some challenges. For future BCTs with similar populations, we would consider following the in-person session with additional shorter face-to-face meetings, rather than phone calls, to refine messages and materials. In addition, from a project management perspective, the team faced time constraints when developing materials since mail delivery time needed to be accounted for.

\section{Limitations}

Our study had a few limitations. First, the number of BCT participants was relatively small $(\mathrm{n}=25)$ and limited to urban Latinos in southern California and may not be generalizable to the overall Latino population. However, participant size was typical for BCT and the goal was to refine materials and to test their effectiveness in future study steps. Second, despite best efforts to recruit Latino patients 
only, 2 participants in the English-speaking BCT were non-Latino. This oversight was discovered during the in-person session, but the research team allowed the individuals to participate given their interest and effort made to attend the meeting. Since the BCT approach is designed to ensure that we hear from all participants, we used this opportunity to further the dialog relevant to the Latino population. Many of our findings were similar across the English- and Spanish-speaking sessions, so we do not believe these individuals' levels of participation negatively impacted our results.

\section{Future Research}

Next steps for the PROMPT study include a comparative effectiveness trial in 2 pilot clinics. ${ }^{16}$ Based on BCT input, we defined 3 intervention arms: 1) automated prompts (ie, text alert before FIT mailing followed by 2 automated phone call reminders within 1 week of FIT receipt in the mail), 2) live prompts (ie, live phone call reminders made within 2 weeks after FIT mailing), and 3) combined automated plus live prompts. Pilot outcomes will be published in a companion article. In addition, to continue the patient engagement process, we will convene a patient council that will re-engage select BCT participants to provide ongoing feedback on PROMPT materials and strategies for the main trial set to commence in July 2018.

Overall, BCT provided a novel approach to engage Latino patients and other stakeholders early in the research process, and to create culturally relevant messages and materials for use in the PROMPT pilot study. This patient engagement approach honors the local and cultural aspects of community within the context of evidence-based health care. This project provided valuable insight into Latino patient perceptions around important colon cancer screening messaging, as well as optimal timing and modality of prompts for FIT completion.

\footnotetext{
The authors express their gratitude to the members of the Colorado High Plains Research Network for sharing their Boot Camp Translation experience and expertise, and to the following community health centers for sharing their outreach materials for the BCT in-person meeting: CareOregon (Portland, OR), Community Health Care (Tacoma, WA), HealthPoint (Renton, WA), JPS Health Network (Fort Worth, TX), Multnomah County Health Department (Portland, OR), San Francisco Health Network (San Francisco, CA), and Sea Mar (Seattle, WA).
}

To see this article online, please go to: http://jabfm.org/content/ 32/3/307.full.

\section{References}

1. Siegel RL, Miller KD, Jemal A. Cancer statistics, 2016. CA Cancer J Clin 2016;66:7-30.

2. American Cancer Society. Key statistics for colorectal cancer. 2016. Available from: https://www. cancer.org/cancer/colon-rectal-cancer/about/keystatistics.html. Accessed October 11, 2017.

3. Bibbins-Domingo K, Grossman DC, Curry SJ, et al. Screening for colorectal cancer: US Preventive Services Task Force recommendation statement. JAMA 2016;315:2564-75.

4. American Cancer Society. Cancer Facts \& Figures for Hispanics/Latinos 2015-2017. Atlanta, GA: American Cancer Society; 2015.

5. American Cancer Society. Colorectal Cancer Facts \& Figures 2017-2019. Atlanta, GA: American Cancer Society; 2017.

6. Nath JB, Costigan S, Lin F, Vittinghoff E, Hsia RY. Federally Qualified Health Center Access and Emergency Department Use Among Children. Pediatrics 2016;138(4) pii: e20160479.

7. Robinson CM, Cassells AN, Greene MA, Beach ML, Tobin JN, Dietrich AJ. Barriers to colorectal cancer screening among publicly insured urban women: No knowledge of tests and no clinician recommendation. J Natl Med Assoc 2011;103:746-53.

8. Goodman MJ, Ogdie A, Kanamori MJ, Cañar J, O'Malley AS. Barriers and facilitators of colorectal cancer screening among Mid-Atlantic Latinos: Focus group findings. Ethn Dis 2006;16:255-61.

9. Norman N, Bennett C, Cowart S, et al. Boot camp translation: A method for building a community of solution. J Am Board Fam Med 2013;26:254-63.

10. Coronado GD, Golovaty I, Longton G, Levy L, Jimenez R. Effectiveness of a clinic-based colorectal cancer screening promotion program for underserved Hispanics. Cancer 2011;117:1745-54.

11. Baker DW, Brown T, Buchanan DR, et al. Comparative effectiveness of a multifaceted intervention to improve adherence to annual colorectal cancer screening in community health centers: A randomized clinical trial. JAMA Intern Med 2014;174:1235-41.

12. Goldberg D, Schiff GD, McNutt R, FurumotoDawson A, Hammerman M, Hoffman A. Mailings timed to patients' appointments: A controlled trial of fecal occult blood test cards. Am J Prev Med 2004; 26:431-5.

13. Goldman SN, Liss DT, Brown T, et al. Comparative effectiveness of multifaceted outreach to initiate colorectal cancer screening in community health centers: A randomized controlled trial. J Gen Intern Med 2015;30:1178-84.

14. Gupta S, Halm EA, Rockey DC, et al. Comparative effectiveness of fecal immunochemical test outreach, 
colonoscopy outreach, and usual care for boosting colorectal cancer screening among the underserved: A randomized clinical trial. JAMA Intern Med 2013; 173:1725-32.

15. Davis MM, Freeman M, Shannon J, et al. A systematic review of clinic and community intervention to increase fecal testing for colorectal cancer in rural and low-income populations in the United StatesHow, what and when? BMC Cancer 2018;18:40.

16. Thompson JH, Davis MM, Leo MC, et al. Participatory Research to Advance Colon Cancer Prevention (PROMPT): Study protocol for a pragmatic trial. Contemp Clin Trials 2018;67:11-5.

17. American Academy of Family Physicians. Boot Camp Translation Training Spring 2017. 2017. Available from: http://www.aafp.org/dam/AAFP/ documents/patient_care/nrn/boot-camp-translationtraining.pdf. Accessed October 10, 2017.

18. Westfall JM, Zittleman L, Backlund-Jarquin P. Boot Camp Translation Guidebook. Denver, CO: Colorado Clinical and Translational Sciences Institute; 2014.

19. Allison C, Zittleman L, Ringel M, et al. Translating the medical home into patient-centred language. London J Prim Care (Abingdon) 2014;6:124-30.

20. National Colorectal Cancer Roundtable. Colon cancer screening communications guidebook: Recommended messaging to reach the unscreened. Atlanta, GA: American Cancer Society; 2017.

21. National Colorectal Cancer Roundtable. Hispanics/ Latinos and colorectal cancer companion guide: Tested messages to reach the unscreened. Atlanta, GA: American Cancer Society; 2016. 